\section{Impact of Toxoplasma gondii infection on human health}

\author{
Taibur Rahman*Jerin-E-Gulshan and Atiqur Rahman
}

Laboratory of Infection Biology, Department of Biochemistry and Molecular Biology, University of Dhaka. Dhaka-1000, Bangladesh

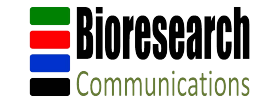

Bioresearch Communications

Volume 8, Issue 1, January 2022

DOI:

https://doi.org/10.3329/brc.v8i1.57049

\begin{abstract}
Toxoplasmosis is one of the most prevalent infectious disease around the globe and it is caused by the parasite named Toxoplasma gondii. Infections normally lead to asymptomatic parasite persistence in immunocompetent warm-blooded hosts, including up to $30-50 \%$ of humans. However, T. gondii infection has also a major medical concern and can lead to life-threatening diseases, after reactivation in immunocompromized individuals, particularly in patients with human immunodeficiency virus/cancer or organ transplant recipients, after vertical transmission to fetuses of pregnant women and by inducing recurrent uveitis in immunocompetent adults. More importantly, T. gondii undergoes stage conversion from its fast-replicating tachyzoite to slow replicating dormant bradyzoite preferentially in the brain and skeletal muscles, and lesser extent in the eye, liver, kidney and lung which enable the parasite to persist for the whole life of an individual. Due to the persistence behavior of the parasite in different parts of human body, T. gondii can develop multiple human diseases with severe clinical symptoms. In this study, we have summarized the association of $T$. gondii in multiple human diseases for instance Encephalitis, Parkinson's disease, Schizophrenia, Heart disease, Ocular Toxoplasmosis, Congenital abnormalities, Cancer and Diabetes. This highlights the potential role of $T$. gondii in developing fatal diseases, particularly in immunocompromised individuals despite having asymptomatic nature of the parasite.
\end{abstract}

KEYWORDS: Toxoplasma gondii, skeletal muscle, brain, immunocompromised, human diseases

RECEIVED: 12 August 2021, ACCEPTED: 30 October 2021

TYPE: Review Article
CORRESPONDING AUTHOR: Dr. Taibur Rahman, Associate Professor, Infection Biology Laboratory, Department of Biochemistry and Molecular Biology, University of Dhaka. Email: taibur@du.ac.bd

\section{Introduction}

Toxoplasmosis, a disease caused by the infection with a protozoan parasite called Toxoplasma gondii (T. gondii), is changing into worldwide jeopardy because the parasite infects $30-50 \%$ of human population worldwide(Jaroslav Flegr et al., 2014). Cat is the definitive host where sexual reproduction of $T$. gondii occurs. Cat can be infected by the predation of previously infected rabbit due to fearless behavior. Following sexual reproduction, $T$. gondii releases into the environment through cat feces as oocysts. These oocysts can persist in the environment for a long time even a year where they can infect intermediate host animals for instance pig, poultry, sheep, goats, cows and birds. In these intermediate hosts animals, T.gondii undergoes stage conversion from its fast replicating tachyzoite into slow replicating bradyzoite preferentially in the brain and skeletal muscles where it establishes chronic infection and act as reservoirs of $T$. gondii transmission into human host. Human can become infected mainly either by eating undercooked/raw meats and meat products or by ingestion of oocysts containing foods, vegetables and water. Congenital transmission and blood transfusion can act as other minor routes of $T$. gondii transmission into human (Figure 1). After primary infection and due to developmental switching of T. gondii in human, the parasite can transmit into different organs of the body for example brain, eyes, skeletal and heart muscles, liver, kidney, testis, etc. Many studies suggest that this asymptomatic infection might also result in the development of other human pathologies particularly in immunocompromised conditions (Sibley, 1995).The clinical spectrum of $T$. gondii infection varies from well to severe general unwellness (Dalimi \& Abdoli, 2012); most typically, infection can result a delicate disease with mild fever and lymphadenopathy. Primary infections with $T$. gondii throughout maternity may cause inborn disease (Desmonts \& Couvreur, 1974). However, some T. gondii infected people develop retinitis that may associate with visual impairments (Commodaro et al., 2009). In immunocompromised patients, $T$. gondii can develop severe neurological problems (HinzeSelch, 2015). Infections with $T$. gondii could manifest carditis with myocardial inflammation and acute heart failure (Alvarado-Esquivel et al., 2016). In rare instances, infection is associated with severe pathological symptoms, together with retinochoroiditis, myocarditis and encephalitis and doubtless resulting in death (Hsu et al., 2014). As it does not show any symptoms normally, it had been long thought that latent $T$. gondii infection was of a very little public health significance except for coinciding immunological disorders, like HIV infection (Lee \& Lee, 2017). 


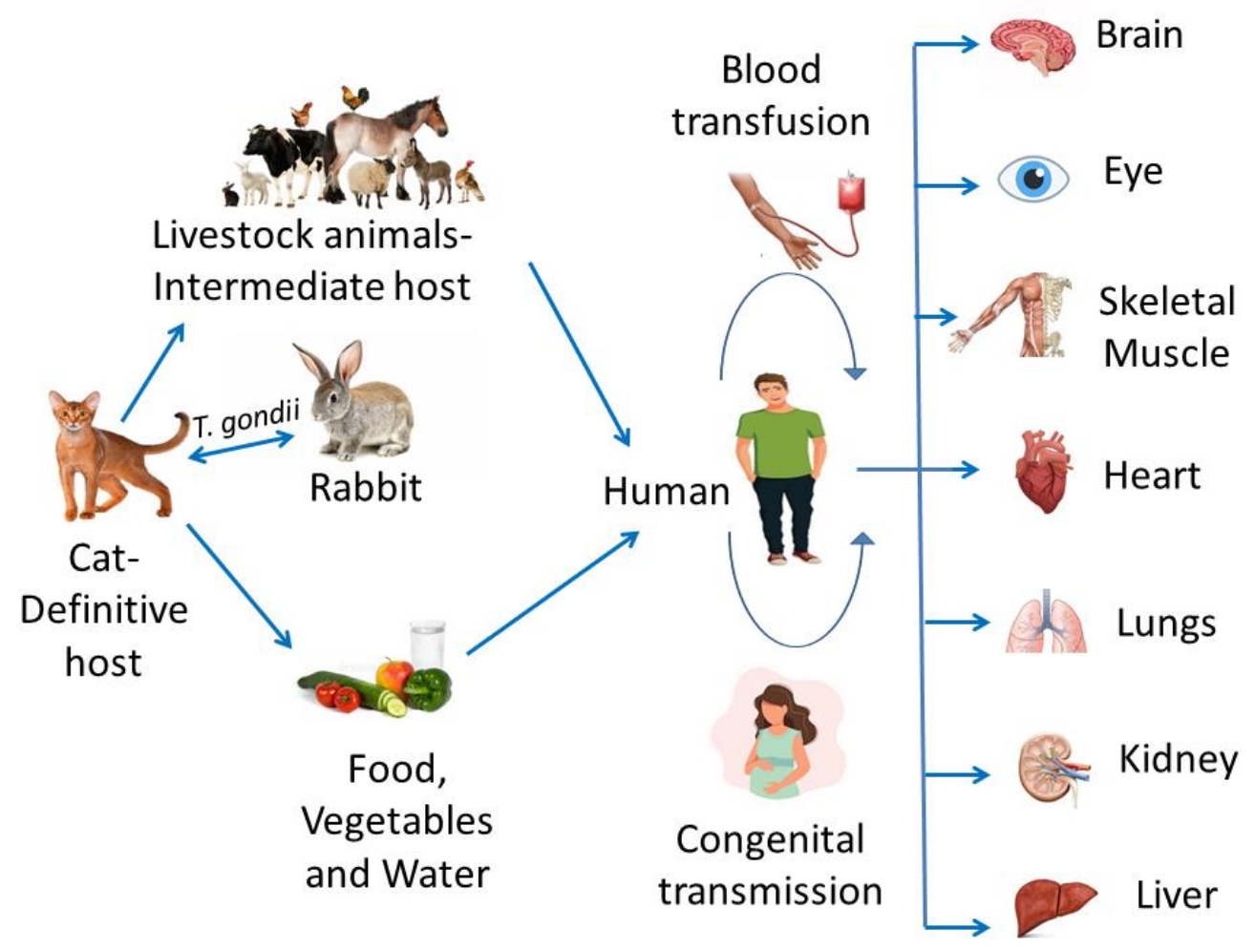

Figure 1. Life cycle and transmission of $T$. gondii into different organs of human body.

The correlation of $T$. gondii with several diseases or severe clinical outcomes has been researched up to this time. In this review, we have summarized $T$. gondii associated human diseases. These includes 1) Encephalitis in AIDS, 2) Parkinson's disease, 3) Depression and suicide, 4) Schizophrenia, 5) heart disease, 6) Ocular Toxoplasmosis, 7) Congenital abnormalities, 8) Epilepsy, 9) Leukemia, 10) Cancer of the oropharynx, 11) Prostate cancer, 12) Asthma 13) Arthritis, 14) Autoimmune disease and 15) Diabetes.

\section{Encephalitis in AIDS}

Toxoplasmic Encephalitis (TE) is an opportunistic lifethreatening chronic infection that affects the central nervous system (CNS) of immunosuppressed individuals such as acquired immune deficiency syndrome (AIDS) patients and transplant recipients (Lee \& Lee, 2017). The reactivation of chronic T. gondii infection is responsible for more than $95 \%$ of TE among AIDS patients where the infection is caused by the progressive loss of cellular immune surveillance. The decline of CD4+ cell count below $100 / \mathrm{mm}^{3}$ in AIDS results in TE (Dannemann et al., 1992). In a study conducted in the US, it was found that 10-40 \%adult AIDS patients were found to have a latent infection with T. gondii and about $33 \%$ of them develop TE (Sibley, 1995). With the much higher occurrence of latent infection the population of Africa, Haiti, Europe and Latin America shows three to four times greater prevalence of TE than the USA. A study in Austria shows 47\% T. gondii specific antibody positive AIDS patients develop TE (Zangerle et al., 1994). One fourth to half of the $T$. gondii infected AIDS patients in Europe and Africa is speculated to develop TE (Clumeck et al., 1984. The epidemiological factors could be possible causes of the increased propensity of TE development in these regions which can be related to the greater load of T.gondii in latent form due to recrudescence of a chronic infection. Only $30-50 \%$ of HIV-positive patients latently infected with $T$. gondii develop TE and the reason is not clear. Certain factors like genetic predisposition, variation in virulence among different strains of the parasite could partially be responsible for the predisposition of the patients to reoccurring infection. Host-genetic factors play important role in developing toxoplasmosis and it was studied using murine model (Suzuki et al., 1991). The population of France, Germany, Central Africa, and Haiti who are HIV positive with CD4 cell counts below $200 / \mathrm{mm}^{3}$ are recommended to undergo several serological testing and detection of T.gondii infection as they have a higher possibility of infection and infection induced TE.

\section{Parkinson's disease}

The development of a long-term neurodegenerative disorder affecting CNS specially hindering movements is called Parkinson disease (PD). The underlying reason for PD is basically unknown; but, progressive impairment of voluntary motor control, which may be a clinical feature of this sickness, is caused by a loss of dopamine neurons of substantia nigra (Michel et al., 2016).A recent study suggests that $T$. gondii tissue cysts are found throughout the whole brain which may cause neurological damage (Boillat et al., 2020). The correlation between T.gondii infection and parkinsonism is not proven yet. According to a finding (Miman et al., 2010) a 
significantly higher rate of anti-T. gondii IgG antibodies are present in Parkinson's patients which were absent in controls. The association of $T$. gondii infection and PD was tested in a group of patients attending public hospitals in Durango City, Mexico (Alvarado-Esquivel et al., 2017). Although the results do not support an association between $T$. gondii infection and PD, however, suggest that $T$. gondii infection might influence on the symptoms of PD. Further research might be needed to understand the association of $T$. gondii and its impact on the development of PD.

\section{Depression and suicide}

One of the leading causes of the global chronic illness burden is depression or major depressive disorder according to the study of WHO on 2013. A mood disorder expressed as few or no interest, severe mood swing, depleted cognitive function, decreased sleep and appetite along with poor energy is termed as Depression (Pratt \& Brody, 2008). It affects an individual physically, personally and professionally. Suicidal tendencies of patients are high and the rate of mortality due to this reason is about $2-8 \%$ and about $50 \%$ of suicidal deaths are due to depression or another psychological disorder (Bachmann, 2018). The underlying reasons for developing major depressive disorder are thought to be genetic, psychological, environmental, family history of psychological disorder, medication on certain drugs, history of trauma or abuse, etc. Depression is not very uncommon in people around the world and it was found to have association with a great extent of mortality to people with T.gondii infection (Duffy et al., 2015). In addition to this, it was shown in a study that $T$.gondii is responsible for bipolar disorder (Hinze-Selch, 2015).

\section{Schizophrenia}

Delusions and hallucinations in adults are found to be associated with some cases of Toxoplasmosis. Early case reports found that the patients with clinical symptoms such as paranoid and bizarre delusions, disorganized speech, flattened affect, auditory and visual hallucinations and thought disorder (Minto \& Roberts, 1959) were initially diagnosed as schizophrenic, and later the patients were found to be diagnosed with $T$. gondii infection suggesting $T$. gondii may contribute to schizophrenia. T.gondii infection can be long term and started at an early life. It can vary from person to person based on genetic predisposition, immunity, dose, time, and virulence of the strain, and the part of the brain affected. There is a fact that T.gondii activates retroviruses in animals (Gazzinelli et al., 1996) and schizophrenic patients had increased retroviral activation within their central nervous systems. Although schizophrenia is expressed after adolescence, it originates at an early stage of brain development. So both prenatal and postnatal infection of T.gondii can result in schizophrenia. Glial cells especially astrocytes are seen to be mostly affected by T.gondii in cell culture (Halonen et al., 1996). Glial abnormalities and a decreased number of astrocytes are seen in Postmortem studies of schizophrenic brains. Schizophrenic persons show abnormalities in neurotransmitters, such as dopamine and norepinephrine, which is later proved to be affected by T.gondii (Conejero-Goldberg et al., 2003).Cognitive function is comparatively poor in T.gondii seropositive schizophrenic patients than seronegative schizophrenic patients of the same age and similar degrees of psychotic symptoms.

\section{Heart Disease}

Chronic heart failure (CHF) involves interactions between the cardiovascular, neuroendocrine and immune systems. In a study, the seropositivity rate for anti-Toxoplasma IgG and IgM antibodies was interrogated by ELISA in patients with CHF ( $\mathrm{n}=97$ ) and control subjects $(\mathrm{n}=50)$ (Yazar et al., 2006). The study found that 66 of 97 (68\%)patients and 18 of 50 (36 $\%$ ) of healthy subjects were found to be positive for IgG antibodies of T.gondii. Therefore, $T$. gondii infection is a risk factor in developing CHF. In addition to this, in a later study indicates that $T$. gondii infection is associated with heart disease (Alvarado-Esquivel et al., 2016). Natural killer cells contribute the first line of non-antigen specific defense against infections.

\section{Ocular Toxoplasmosis}

Toxoplasmosis is an important cause of ocular disease in immunosuppressed as well as immunocompetent persons. The uvea is affected which results in uveitis and it is believed that a significant reason behind this is ocular toxoplasmosis which is caused by T.gondii infection. A large number of ocular cases are secondary to postnatal infection with $T$. gondii. It is observed that $70-90 \%$ of patients with congenital toxoplasmosis and $10-12 \%$ of patients with postnatal infection develop ocular disease (Gilbert et al., 2008). When ocular lesions appear the serum IgG titers may be low and IgM may be absent. The lesions are intensely white, with an overlying vitreous inflammatory response. Current lesions accompanied by a severe vitreous inflammatory reaction might have a "headlight in the fog" appearance. Choroid inflammation is usually secondary and does not occur if retinal inflammation is absent. Severe secondary iridocyclitis can occur as well. Several people with acquired toxoplasmosis may suffer from atypical ocular lesions, vasculitis, vitritis, anterior uveitis, without developing a focal necrotizing retinochoroiditis. Drugs so far developed cannot eliminate tissue cysts and therefore cannot prevent chronic infection. A toxoplasmosis scar will be related to severe visual field loss once it happens near to the optic disc (Harrell \& Carvounis, 2014).Ocular infection has long been considered a sickness that was primarily caused by inborn infection, while symptomatic ocular caused by acquired infection following birth was considered rare. This finding was confirmed by different teams in most populations exposed to T. gondii (Atmaca et al., 2004; Bosch-Driessen et al., 2002). The rate of congenital infection varies from $0.13 \%$ to $0.01 \%$ and it depends on the geographical region of the world. In neonates with inborn infection, the incidence of retinochoroiditis varies in numerous studies however is also as high as $80 \%$ (Vasconcelos-Santos et al., 2009). Infants are mostly infected by ocular lesion which is the most general instance of ingrained infection. Ocular infection typically presents with classic ophthalmic findings, and therefore the diagnosing is reached by clinical examination with none laboratory confirmation of T.gondii infection (Dodds, 2006). Vision defect is also secondary to a macular lesion, whereas lesions settled at the peripheral tissue layer typically cause vision loss secondary to severe vitreous inflammation (Mets et al., 1996). Nervus opticus involvement is less common however might induce severe visual field defects in addition to the loss of visual sense. 


\section{Congenital abnormalities}

Congenital toxoplasmosis can cause a wide spectrum of clinical manifestations; however, it does not produce definite symptoms in $75 \%$ of newborns. Interestingly, about 63 years ago, it was observed that mother of Down syndrome affected children had a much greater probability of having latent toxoplasmosis $(84 \%)$ than the mother of normal children (32\%); however, the probability of infection in fathers did not alter between children with and without this disorder. The membrane of the surface of T.gondii has distinct protein transporters which bind to folic acid with high affinity and it results in accession and salvage of exogenous folate compound (Massimine et al., 2005). Folate deficiency occurs as a result of this phenomenon. Iron and deficiencies are also caused which hampers the developing fetus and it is followed by T.gondii infection. In a study in Czech Republic, the development of embryos in mothers with latent toxoplasmosis was slower compared to those who did not have an infection, although, the birth weight of newborns was approximately the same as those of infection-free mothers (Kanková \& Flegr, 2007).There is a possibility that the effect of toxoplasmosis on the development of embryos is relatively different in other parts of the world, however further studies are needed to address this.

\section{Epilepsy}

Epilepsy is a neurological disorder characterized by abnormal brain activity, causing seizures, sensations, periods of unusual behaviour, and sometimes experiencing consciousness. The association between latent toxoplasmosis and cryptic epilepsy has already been suggested to exist based on case control studies (Yazar et al., 2003), implying a strong correlation between epilepsy and $T$. gondii infection. Although these studies suggest a correlation another study contradicts the finding (Critchley et al., 1982).

\section{Leukemia}

A meta-analysis performed in 2016, suggests a possible link of T. gondii infection with the occurrence of leukemia (Huang et al., 2016). This link was further evidenced by another study where it was shown that $T$. gondii specific IgG was detected in $96(56.4 \%)$ leukemia patients and $72(42.4 \%)$ healthy subjects while $\operatorname{IgM}$ antibodies were detected in 10 patients $(5.9 \%)$ and $3(1.8 \%)$ controls (Huang et al., 2014). In a recent study performed on a relatively higher number of sample sizes, it was found that $T$. gondii infection in leukemic children is significantly higher compared to healthy children(Na Zhou et al., 2019). Although studies suggest a possible link between $T$. gondii infection and leukemia, however, the cause and effect relationship amongst them has not established; $T$. gondii could be a risk factor of developing leukemia or patients may develop a secondary infection as a result of immunosuppression. Further large-scale studies may warrant to address this.

\section{Cancer of the mouth/oropharynx}

Tonsillitis is a common manifestation of acute toxoplasmosis which can develop local chronic inflammation resulting in precancerous changes in patients An association of the prevalence of toxoplasmosis with oral cancer has been reported in 2018(N Zhou et al., 2018). This study showed oral cancer patients were reported to have significantly higher $T$. gondii specific antibodies ( $\operatorname{IgG}$ or $\operatorname{IgM})$ than healthy subjects.

\section{Prostate cancer}

Male rats infected with the parasite have increased level of testosterone. It can be correlated with higher cases of prostate cancer and hypertrophy in human (J. Flegr et al., 2008; Lim et al., 2013). Prostate gland and seminal vesicle inflammation are noted in the histopathologic studies of the male reproductive system in sheep which were infected with T.gondii (Lopes et al., 2011).In mouse model, T. gondii infection was found to induce a chronic inflammatory reaction in the prostate, leading to microglandular hyperplasia in the inflamed prostates, suggesting a role of $T$. gondii as a risk factor of prostate cancer.

\section{Asthma}

Toxoplasmosis has a negative correlation with asthma occurrence. It may be possible because of the antiinflammatory effect of histamine. Patients with asthma produce a large amount of histamine which inflect the balance of cytokine TH1/TH2(Packard \& Khan, 2003), thus exerting beneficial anti-inflammatory effects. While the opposite effect is observed in $T$. gondii infection that preferentially generates Th1 response and therefore, this may explain a potential negative correlation of $T$. gondii infection and the occurrence of asthma.

\section{Arthritis}

T. gondii can be associated with the development of arthritis which is supported by a number of studies. The association of T. gondii infection with arthritis is evident from a case-control study in the Chinese population $(n=820$, for both patients and controls), where $T$. gondii specific antibodies were measured by ELISA(Tian et al., 2017) and the abundance of IgG of the respected antibody was found to be much greater in patients suffering from arthritis (18.8\%). It was only $12 \%$ in case of healthy individuals. Patients with Rheumatoid arthritis and reactive arthritis has greater infection seropositivity with T.gondii than osteoarthritis, infectious and gouty arthritis. Another study on Egyptian rheumatoid arthritis patients found a positive correlation of seropositivity of $T$. gondii not only on the occurrence of the disease but also on intensified clinical characteristics, including longer disease duration, higher number of tender and swollen joints, and longer time morning stiffness (El-Henawy et al., 2017).

\section{Autoimmune disease}

Autoimmune diseases are diverse clinical conditions, where the immune system acts on self-antigen. The prevalence of $T$. gondii-specific antibodies was reported to be significantly higher in systemic sclerosis $(\mathrm{p}<0.0001)$, thyroid autoimmune diseases $(\mathrm{p}<0.0001)$, pemphigus vulgaris $(\mathrm{p}<0.0001)$ and vasculitis compared to control subjects (Petrikova et al., 2010). However, this was unchanged in polymyositis, ChurgStrauss vasculitis, SLE, multiple sclerosis etc. So, it can be concluded that T.gondii has an association with several, but not all, autoimmune diseases.

\section{Diabetes}


Multiple case-control and meta-analysis suggest a positive mellitus) and thus toxoplasmosis is considered as a potential risk factor for this disease. However, no statistically significant association was observed between $T$. gondiiand type 1 DM. Importantly, a systemic review and meta-analysis carried out on 4072 subjects from ten studies reported a positive correlation of $T$. gondii infection and type $2 \mathrm{DM}(\mathrm{Li}$ et al., 2018). The authors reported that 7 out of 10 publications showed a positive correlation and overall, they reported a significantly higher prevalence of $T$. gondii in type $2 \mathrm{DM}$ patients $(47.8 \%$; range $6.4-65.1 \%)$ than healthy subjects (25.9\%; range 3.2-59.0\%) (Li et al., 2018).

\section{Conclusion}

T. gondii is a widely distributed parasite in nature and therefore the infection rate is very high in human population worldwide. Due to the persistent nature of the parasite, $T$. gondii can be associated with multiple human diseases. In this study, we have summarized the impact $T$. gondii in various human diseases i.e encephalitis, Parkinson's disease, Schizophrenia, Heart disease, Ocular Toxoplasmosis, Congenital abnormalities, Cancer and Diabetes etc. Understanding the association of $T$. gondii in human diseases would be beneficial for better management and treatment of $T$. gondii mediated diseases.

\section{Acknowledgements}

The authors thank Department of Biochemistry and Molecular Biology, University of Dhaka and Ministry of Education, Bangladesh for their cooperation.

\section{Conflict of Interest}

The authors declare no conflicts of interest regarding the publication of this article.

\section{References}

1. Alvarado-Esquivel, C., Méndez-Hernández, E. M., SalasPacheco, J. M., Ruano-Calderón, L. Á., HernándezTinoco, J., Arias-Carrión, O., Sánchez-Anguiano, L. F., Castellanos-Juárez, F. X., Sandoval-Carrillo, A. A., Liesenfeld, O., \& Ramos-Nevárez, A. (2017). Toxoplasma gondii exposure and Parkinson's disease: A case-control study. BMJ Open, 7(2), 1-7. https://doi.org/10.1136/bmjopen-2016-013019

2. Alvarado-Esquivel, C., Salcedo-Jaquez, M., SanchezAnguiano, L. F., Hernandez-Tinoco, J., Rabago-Sanchez, E., Beristain-Garcia, I., Liesenfeld, O., Estrada-Martinez, S., Perez-Alamos, A. R., \& Alvarado-Soto, E. (2016). Association Between Toxoplasma gondii Exposure and Heart Disease: A Case-Control Study. Journal of Clinical Medicine Research, 8(5), 402-409. https://doi.org/10.14740/jocmr2525w

3. Atmaca, L. S., Simsek, T., \& Batioglu, F. (2004). Clinical features and prognosis in ocular toxoplasmosis. Japanese Journal of Ophthalmology, 48(4), 386-391. https://doi.org/10.1007/s10384-003-0069-0

4. Bachmann, S. (2018). Epidemiology of suicide and the psychiatric perspective. International Journal of Environmental Research and Public Health, 15(7), 1-23. https://doi.org/10.3390/ijerph15071425 correlation of chronic toxoplasmosis with type 2DM (diabetes

5. Boillat, M., Hammoudi, P. M., Dogga, S. K., Pagès, S., Goubran, M., Rodriguez, I., \& Soldati-Favre, D. (2020). Neuroinflammation-Associated Aspecific Manipulation of Mouse Predator Fear by Toxoplasma gondii. Cell Reports, $30(2)$, https://doi.org/10.1016/j.celrep.2019.12.019

320-334.e6.

6. Bosch-Driessen, L. E. H., Berendschot, T. T. J. M., Ongkosuwito, J. V, \& Rothova, A. (2002). Ocular toxoplasmosis: clinical features and prognosis of 154 patients. Ophthalmology, 109(5), 869-878. https://doi.org/10.1016/s0161-6420(02)00990-9

7. Commodaro, A. G., Belfort, R. N., Rizzo, L. V., Muccioli, C., Silveira, C., Burnier, M. N., \& Belfort, R. (2009). Ocular toxoplasmosis - An update and review of the literature. Memorias Do Instituto Oswaldo Cruz, 104(2), 345-350. 02762009000200030 https://doi.org/10.1590/S0074-

8. Conejero-Goldberg, C., Torrey, E. F., \& Yolken, R. H. (2003). Herpesviruses and Toxoplasma gondii in orbital frontal cortex of psychiatric patients. Schizophrenia Research, 60(1), 65-69. https://doi.org/10.1016/s09209964(02)00160-3

9. Critchley, E. M., Vakil, S. D., Hutchinson, D. N., \& Taylor, P. (1982). Toxoplasma, Toxocara, and epilepsy. Epilepsia, 23(3), 315-321. https://doi.org/10.1111/j.15281157.1982.tb06197.x

10. Dalimi, A., \& Abdoli, A. (2012). Latent Toxoplasmosis and Human. Iranian J Parasitol, 7(1), 1-17. http://ijpa.tums.ac.ir

11. Dannemann, B., McCutchan, J. A., Israelski, D., Antoniskis, D., Leport, C., Luft, B., Nussbaum, J., Clumeck, N., Morlat, P., Chiu, J., Vilde, J. L., Orellana, M., Feigal, D., Bartok, A., Heseltine, P., Leedom, J., \& Remington, J. (1992). Treatment of toxoplasmic encephalitis in patients with AIDS: A randomized trial comparing pyrimethamine plus clindamycin to pyrimethamine plus sulfadiazine. Annals of Internal Medicine, 116(1), 33-43. https://doi.org/10.7326/00034819-116-1-33

12. Desmonts, G., \& Couvreur, J. (1974). Toxoplasmosis in pregnancy and its transmission to the fetus. Bulletin of the New York Academy of Medicine: Journal of Urban Health, 50(2), 146-159.

13. Dodds, E. M. (2006). Toxoplasmosis. Current Opinion in Ophthalmology, 17(6), 557-561. https://doi.org/10.1097/ICU.0b013e32801094ca

14. Duffy, A. R., Beckie, T. M., Brenner, L. A., Beckstead, J. W., Seyfang, A., Postolache, T. T., \& Groer, M. W. (2015). Relationship Between Toxoplasma gondii and Mood Disturbance in Women Veterans. Military Medicine, 180(6), 621-625. https://doi.org/10.7205/milmed-d-1400488

15. El-Henawy, A. A., Hafez, E. A. R., Nabih, N., Shalaby, N. M., \& Mashaly, M. (2017). Anti-Toxoplasma antibodies in Egyptian rheumatoid arthritis patients. Rheumatology International, 37(5), 785-790. https://doi.org/10.1007/s00296-017-3703-8

16. Flegr, J., Lindová, J., \& Kodym, P. (2008). Sex-dependent toxoplasmosis-associated differences in testosterone concentration in humans. Parasitology, 135(4), 427-431. https://doi.org/10.1017/S0031182007004064 
17. Flegr, Jaroslav, Prandota, J., Sovičková, M., \& Israili, Z. H. (2014). Toxoplasmosis - A global threat. Correlation of latent toxoplasmosis with specific disease burden in a set of 88 countries. PLoS ONE, 9(3), e90203. https://doi.org/10.1371/journal.pone.0090203

18. Gazzinelli, R. T., Sher, A., Cheever, A., Gerstberger, S., Martin, M. A., \& Dickie, P. (1996). Infection of human immunodeficiency virus 1 transgenic mice with Toxoplasma gondii stimulates proviral transcription in macrophages in vivo. The Journal of Experimental Medicine, 183(4), 1645-1655. https://doi.org/10.1084/jem.183.4.1645

19. Gilbert, R. E., Freeman, K., Lago, E. G., Bahia-Oliveira, L. M. G., Tan, H. K., Wallon, M., Buffolano, W., Stanford, M. R., Petersen, E., Thulliez, P., Rommand, S., Peyron, F., Schmidt, D. R., Paul, M., Prusa, A., Hayde, M., Pollak, A., Bessieres, M. H., Franck, J., ... Cortina-Borja, M. (2008). Ocular sequelae of congenital toxoplasmosis in Brazil compared with Europe. PLoS Neglected Tropical Diseases, 2(8), 8-14.

https://doi.org/10.1371/journal.pntd.0000277

20. Halonen, S. K., Lyman, W. D., \& Chiu, F. C. (1996). Growth and development of Toxoplasma gondii in human neurons and astrocytes. Journal of Neuropathology and Experimental Neurology, 55(11), 1150-1156. https://doi.org/10.1097/00005072-199611000-00006

21. Harrell, M., \& Carvounis, P. E. (2014). Current treatment of toxoplasma retinochoroiditis: an evidence-based review. Journal of Ophthalmology, 2014, 273506. https://doi.org/10.1155/2014/273506

22. Hinze-Selch, D. (2015). Toxoplasma gondii infection and neuropsychiatric disease: current insight. Reports in Parasitology, 43. https://doi.org/10.2147/rip.s52980

23. Hsu, P. C., Groer, M., \& Beckie, T. (2014). New findings: Depression, suicide, and Toxoplasma gondii infection. Journal of the American Association of Nurse Practitioners, 26(11), 629-637.

https://doi.org/10.1002/2327-6924.12129

24. Huang, Y., Huang, Y., Chang, A., Wang, J., Zeng, X., \& Wu, J. (2016). Is Toxoplasma Gondii Infection a Risk Factor for Leukemia? An Evidence-Based Meta-Analysis. Medical Science Monitor: International Medical Journal of Experimental and Clinical Research, 22, 1547-1552. https://doi.org/10.12659/msm.897155

25. Huang, Y., Xie, D., Tang, N., Wang, J., Zeng, X., Zhao, P., \& He, L. (2014). XRCC1 Arg399Gln variation and leukemia susceptibility: evidence from 2,647 cases and 5,518 controls. Tumour Biology: The Journal of the International Society for Oncodevelopmental Biology and Medicine, 35(1), 799-808. https://doi.org/10.1007/s13277013-1110-7

26. Kanková, S., \& Flegr, J. (2007). Longer pregnancy and slower fetal development in women with latent "asymptomatic" toxoplasmosis. BMC Infectious Diseases, 7, 114. https://doi.org/10.1186/1471-2334-7-114

27. Lee, S.-B., \& Lee, T.-G. (2017). Toxoplasmic Encephalitis in Patient with Acquired Immunodeficiency Syndrome. Brain Tumor Research and Treatment, 5(1), 34. https://doi.org/10.14791/btrt.2017.5.1.34

28. Li, Y.-X., Xin, H., Zhang, X.-Y., Wei, C.-Y., Duan, Y.-H., Wang, H.-F., \& Niu, H.-T. (2018). Toxoplasma gondii Infection in Diabetes Mellitus Patients in China:
Seroprevalence, Risk Factors, and Case-Control Studies. BioMed Research International, 2018, 4723739. https://doi.org/10.1155/2018/4723739

29. Lim, A., Kumar, V., Hari Dass, S. A., \& Vyas, A. (2013). Toxoplasma gondii infection enhances testicular steroidogenesis in rats. Molecular Ecology, 22(1), 102110. https://doi.org/10.1111/mec.12042

30. Lopes, W. D. Z., Santos, T. R., Luvizotto, M. C. R., Sakamoto, C. A. M., Oliveira, G. P., \& Costa, A. J. (2011). Histopathology of the reproductive system of male sheep experimentally infected with Toxoplasma gondii. Parasitology Research, 109(2), 405-409. https://doi.org/10.1007/s00436-011-2268-9

31. Massimine, K. M., Doan, L. T., Atreya, C. A., Stedman, T. T., Anderson, K. S., Joiner, K. A., \& Coppens, I. (2005). Toxoplasma gondii is capable of exogenous folate transport. A likely expansion of the BT1 family of transmembrane proteins. Molecular and Biochemical Parasitology, 144(1), 44-54.

https://doi.org/10.1016/j.molbiopara.2005.07.006

32. Mets, M. B., Holfels, E., Boyer, K. M., Swisher, C. N., Roizen, N., Stein, L., Stein, M., Hopkins, J., Withers, S., Mack, D., Luciano, R., Patel, D., Remington, J. S., Meier, P., \& McLeod, R. (1996). Eye manifestations of congenital toxoplasmosis. American Journal of Ophthalmology, 122(3), 309-324. https://doi.org/10.1016/s00029394(14)72057-4

33. Michel, P. P., Hirsch, E. C., \& Hunot, S. (2016). Understanding Dopaminergic Cell Death Pathways in Parkinson Disease. Neuron, 90(4), 675-691. https://doi.org/10.1016/j.neuron.2016.03.038

34. Miman, O., Kusbeci, O. Y., Aktepe, O. C., \& Cetinkaya, Z. (2010). The probable relation between Toxoplasma gondii and Parkinson's disease. Neuroscience Letters, 475(3), 129-131. https://doi.org/10.1016/j.neulet.2010.03.057

35. Minto, A., \& Roberts, F. J. (1959). The psychiatric complications of toxoplasmosis. Lancet (London, England), 1(7084), 1180-1182. https://doi.org/10.1016/s0140-6736(59)91187-0

36. Packard, K. A., \& Khan, M. M. (2003). Effects of histamine on Th1/Th2 cytokine balance. International Immunopharmacology, 3(7), 909-920. https://doi.org/10.1016/S1567-5769(02)00235-7

37. Petrikova, J., Agmon-Levin, N., Shapira, Y., Barzilai, O., Ram, M., Gilburd, B., Selmi, C., Nicola, B., Larida, B., Gershwin, M. E., Valentini, G., Matucci-Cerini, M., Anaja, J.-M., Katz, B.-S. P., Blank, M., \& Shoenfeld, Y. (2010). Prevalence of Toxoplasma antibodies among patients with various autoimmune diseases. Annals of the Rheumatic Diseases, 69(Suppl $\left.{ }^{2}\right), \quad$ A8-A8. https://doi.org/10.1136/ard.2010.129577t

38. Pratt, L. A., \& Brody, D. J. (2008). Depression in the United States household population, 2005-2006. NCHS Data Brief, 7, 1-8.

39. Sibley, D. K. H. and L. D. (1995). Toxoplasma gondii Comprises Three Clonal Lineages: Correlation of Parasite Genotype with Human Disease Author (s): Daniel K. Howe and L. David Sibley Published by: Oxford University Press Stable

URL: http://www.jstor.org/stable/30134669 Accessed: Journal of Infectious Diseases, 172(6), 1561-1566. 
40. Suzuki, Y., Joh, K., Orellana, M. A., Conley, F. K., \& Remington, J. S. (1991). A gene(s) within the H-2D region determines the development of toxoplasmic encephalitis in mice. Immunology, 74(4), 732-739.

41. Tian, A.-L., Gu, Y.-L., Zhou, N., Cong, W., Li, G.-X., Elsheikha, H. M., \& Zhu, X.-Q. (2017). Seroprevalence of Toxoplasma gondii infection in arthritis patients in eastern China. Infectious Diseases of Poverty, 6(1), 153. https://doi.org/10.1186/s40249-017-0367-2

42. Vasconcelos-Santos, D. V., Machado Azevedo, D. O., Campos, W. R., Oréfice, F., Queiroz-Andrade, G. M., Carellos, E. V. M., Castro Romanelli, R. M., Januário, J. N., Resende, L. M., Martins-Filho, O. A., de Aguiar Vasconcelos Carneiro, A. C., Almeida Vitor, R. W., \& Caiaffa, W. T. (2009). Congenital toxoplasmosis in southeastern Brazil: results of early ophthalmologic examination of a large cohort of neonates. Ophthalmology, 116(11), 2199-205.e1. https://doi.org/10.1016/j.ophtha.2009.04.042

43. Yazar, S., Arman, F., Yalçin, S., Demirtaş, F., Yaman, O., \& Sahin, I. (2003). Investigation of probable relationship between Toxoplasma gondii and cryptogenic epilepsy. Seizure, 12(2), 107-109. https://doi.org/10.1016/s10591311(02)00256-X
44. Yazar, S., Gur, M., Ozdogru, I., Yaman, O., Oguzhan, A., \& Sahin, I. (2006). Anti-Toxoplasma gondii antibodies in patients with chronic heart failure. Journal of Medical Microbiology, 55(1), 89-92. https://doi.org/10.1099/jmm.0.46255-0

45. Zangerle, R., Allerberger, F., Israelski, D., Phair, J., \& Remington, J. S. (1994). High risk of developing toxoplasmic encephalitis in AIDS patients seropositive to Toxoplasma gondii. Journal of Acquired Immune Deficiency Syndromes, 7(2), 207-208.

46. Zhou, N, Zhang, X. Y., Li, Y. X., Wang, L., Wang, L. L., \& Cong, W. (2018). Seroprevalence and risk factors of Toxoplasma gondii infection in oral cancer patients in China: a case-control prospective study. Epidemiology and Infection, 146(15), 1891-1895. https://doi.org/10.1017/S0950268818001978

47. Zhou, Na, Fu, H., Wang, Z., Shi, H., Yu, Y., Qu, T., Wang, L., Zhang, X., \& Wang, L. (2019). Seroprevalence and risk factors of Toxoplasma gondii infection in children with leukemia in Shandong Province, Eastern China: a case-control prospective study. PeerJ, 7, e6604. https://doi.org/10.7717/peerj.6604 